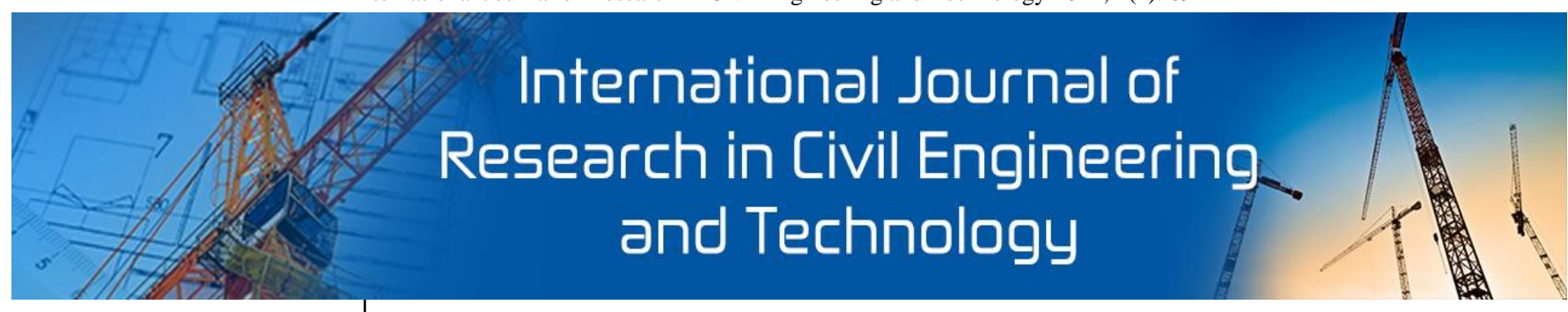

E-ISSN: 2707-8272

P-ISSN: 2707-8264

IJRCET 2021; 2(2): 09-12

Received: 07-06-2021

Accepted: 19-07-2021

Yangka Rigyal

College of Science and

Technology, Rinchending,

Phuentsholing, Chukkha, Bhutan
Corresponding Author: Yangka Rigyal College of Science and Technology, Rinchending, Phuentsholing, Chukkha, Bhutan

\section{A model for integrating construction design and data}

\section{Yangka Rigyal}

DOI: https://doi.org/10.22271/27078264.2021.v2.i2a.16

\begin{abstract}
The strain of timely retrieval of useful information from various data sources is a major cause of low productivity in the construction industry. The box of this examination is to afford a new methodology to holder the data-heterogeneity problems arise upon in a construction change. This enquiry proposes a tree-structured product model, which binds design data, cost data and list data together, as a practical solution for the data integration difficult in formation ventures. The style uses the knowledge representation of manufacturing projects based on an ontology. Metadata are charity to describe the concrete structure of the mission knowledge. The impression of views is used to answer particular queries from different domains. A general approach is under development to maintenance queries about a certain assembly project from different user perspectives based on diverse erection records sources in a dynamic environment. The research is composed of two major parts: (1) unite the assorted construction data into a tree structure; and (2) retrieve information also acquire domain views by specifying the ways of traversing the tree. The future goal is to advance a prototype that will support some of the most main occupations needed in today's construction projects. Functional illustrations are used to determine the validity of the anticipated association.
\end{abstract}

Keywords: metadata perspectives; ventures

\section{Introduction}

Weighty improvements in productivity have been completed in the manufacturing industry through the application of information amalgamation tools, such as CAD/CAM, in their daily operations. However, in the assembly industry, the improvement has been quite limited even though CAD, scheduling and estimating software abstain been widely used in construction for quite a long time. Various reasons devise been identified for this discrepancy - an important one is the lack of information integration across the continuous participants in construction projects. Truly, overweight 60-70 percent of the in drudgery hours of a broad contractor's staff can be spent clarifying and confirming design or construction information (Nambayashi et al. 2000) ${ }^{[1]}$. Marine associations are import facilities erected and committed in shoreline boundaries or open mountains for the employment of various aquatic wealth and the safeguarding of its continuous operations. Commonly, the marine edifices can be on bad terms into three types: fixed, movable (or floating structures) and free of charge schedules. Motionless activities are fixed on the seabed on a durable basis by using mounds or the gravity of productions.

Even though the design, cost estimating and project scheduling processes are computerized individually, the information retrieval status quo does not improve much because each application is using its own data model. The high total associated with data searching and retrieval is one of the causes associated with the low throughput of the construction industry. In the widely held of current construction projects, CAD systems are still used only as the illustration tool for the architect, instead of being used as a unified design information database. As a result, mark retrieval has to be done manually in spite of its electronic plan. In a emblematic scenario, when engineers, venture officers, estimators or even other architects want to retrieve policy information from the drawings, they might have to search tons, if not hundreds of documents to find the scattered pieces and put them together. The information search and retrieval route becomes an extremely time consuming job, mainly for large and complex projects. Moreover, this guide salvage process is error prone. It has been estimated that up to $50 \%$ of all production changes in the business industry are adjustments of errors rather than changed requirements or real improvements (Engelke 1987). Considering the fragmented setting of the construction industry, the error rate is most prospective difficult than that in the manufacturing industry. 
Even still the design, cost estimating and project scheduling progressions are computerized individually, the data retrieval situation does not improve much because each one tender is using its own data model. The high cost add-on with information searching and retrieval is one of the bases associated with the low productivity of the construction engineering. With the increased use of erection software, the traditional cross-disciplinary communication delinquent is becoming increasingly an issue of records exchange and data sharing between different software applications. Unfortunately, most of the software packages used in the erection industry have their own proprietary data plan, for the reason that they were developed by different vendors. Several international standardization efforts, together with STEP (Standard for the Exchange of Product classic data) and IFC (the Industry Foundation Classes), have been carry out to address this communication problem, conversely, the results have been mixed so far. A common data standard does not, in itself, provide the necessary funds on the way to integrate information and provide views useful to humans. As of this spell, the penalty area of this homework be placed propose and develop a new data amalgamation model used for construction that to some degree outspreads our current knacks to provide relevant statistics to decision draftswomen.

\section{Background}

Assistants have considered at common product and/or process models as the solution to the hitches caused by fragmented construction documents. Many copies drink been proposed in trying to address construction data amalgamation issues by both industry and university circles. Initially, these attempts could be divided into two (paired) categories: (1) the standard (product and/or process) archetypal; and (2) the Integrated Project Database approach. Both categories are discussed in detail below. Standardization involves all applications to use the same data model to explain the communication problem among the different domain tenders, thus allowing all the applications to be able to discussion data with each other. Alternatively, it requires those tenders to at least support a neutral data format as a mediocre for the data exchange. This neutral data format is an assimilated data model, which captures the full semantics of an office block system and its components. In this methodology, each application only works with a subset of the model. This subset is habitually designated as a part model. Data discussion jerry can be achieved between all the aspect models by mapping concluded the integrated data model. There are two international tuning efforts that address the illustration of building designs: STEP being developed by the Universal Organization for Adjustment; and IFC, which are specifications for a outmoded of identical object def- A being developed by the Universal Alliance for Interoperability (IAI). Both standards define static house photographs aimed at the transfer of data between submissions. In addition to the worldwide tuning efforts, there exist several local standardization efforts, for example, the RATAS model (Enkovaara et al. 1988) ${ }^{[7]}$, the Unified Data Model of the COMBINE project (Sun and Lockley 1997) ${ }^{[10]}$, and the Logical Range Model for CIM Steel (2000). All these copies have adopted an object-oriented paradigm, which describes the building system using objects or entities and their inter-relationships and they are moreover compliant with the STEP or IFC data representations.

\section{Integrated project database approach}

This line integrates AEC bids through an implemented project database. The aim of a Unified Project Database is to provide consistent and unswerving loading of the project information, and to serve as a data discussion spoke for different tasks during the construction process. A case in point of this approach is Gallicon (Sun et al. 2000) ${ }^{[3]}$. One delinquent with both approaches is that these demonstrations lack extensibility. They are restricted by a predefined semantic demonstration. Any changes to the predefined demonstration entail the update of the whole system. In this sense, these structures are tightly coupled. Cases may occur over time in which changes are needed or some apparatuses may need to be further to the representation to adapt it to some actual context. Thus, the predefined schema approach may be useful in a all-purpose sense, but possibly will be hard to handle in every particular situations (O'Brien et al. 2002). Another problem is that some of these representations (like RATAS) do not funding multiple views from different domains because of their rigid depiction chucks (Rivard and Fenves 2000) ${ }^{[9]}$

\section{Research methodology}

Due to the want of real data relating on the way to quality in the Jordanian housing projects, the inventers use an exploratory method to categorize the most related value factors. The present aim of this study was well-known: factors disturbing quality in the Jordanian housing sector and their relative importance. This exploratory research includes two segments of extensive review of the literature and a survey questionnaire by personal interviews in the housing part in Jordan. The examination design started with divorcing a research problem, and then it provided a review of the available literature in order to understand previous research in eminence and relevant fields. This has led to a determination of the key aim and objectives, and then providing the initial concrete framework for quality factors. The survey was conducted by means of structured personal interviews indoors two months (September and October 2013), supported out face to face for read-through the information accuracy, and developed an understanding of quality factors based on the designed questionnaire. For the duration of the discussions, interviewees be situated briefed on research problem, research objectives and quality factors. The interviews were conducted at the properties of the number of companies that agreed to participate. The opinion poll were answered by free-lancers and architects who be located in charge of quality awareness to ensure that the respondent has the necessary data to respond. All the companies included in the survey were located in Amman due the constraints of time and cost. Amman is considered to be the main pecuniary spoke of the kingdom, and 77 percent of the housing projects are concentrated there (JEA, 2013). Supreme of the survey questions were adopted from previous literature that had been used to limit the factors distressing quality in construction. The questionnaire be situated made of two parts: the demographic evidence of accused and quality factors. 


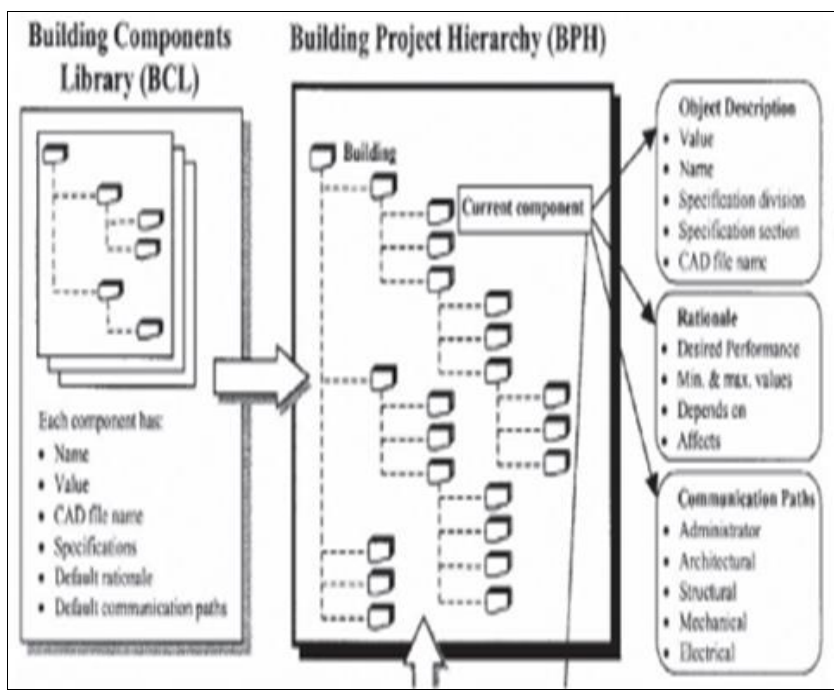

Fig 1: Methodology

The first part, the $\mathrm{BPH}$, stores all erection data and represents this data as a hierarchy of full of life objects. Each object has information about its values, official papers, design rationale, and communication paths. The second part, the BCL, is a central repository of common shop components that allows a building project hierarchy to be designated with little effort. The third part, the DCM trials, manages the design changes made to any object in the BPH and preserves track of the history of changes made by all disciplines. This data model provides a good reference on behalf of the proposed model. But there are also problems thru this model: (1) the BPH and BCL do not link to the 3D CAD piece, instead they link to the $\mathrm{CAD}$ document, which makes the aforementioned difficult in practice to check the consistency and laying-off between the design factor and the BCL; and (2) because only a small portion of the statistics about each domain needs to be exchanged for design matching, the four central databases, one for each professional (architect, structural engineer, mechanical engineer plus electrical engineer), may store a lot of information that does not need en route for be exchanged. This model could be located further developed by linking to the true bits and pieces in the CAD tool.

\section{Virtual product model}

The Practical Product Archetypal (Clayton et al. 1996) [6] supports early stage introductory intangible design. It differs from the general object-oriented copies in that it maps the obviously represented semantic and striking entities rather than unifying the two. The system is unruffled of four modules: form; function; behavior; and rational. It uses an isolated representational model to epitomize the architectural policy design in 3D. The emblematic model is engendered after the cool establishes the 3D drawing by interactively conveying $3 \mathrm{D}$ forms and function specifications to the symbolic knobs. The cerebral segment afterwards uses the noted symbolic section to predict the behavior of the design. This attitude avoids the stiffness of the predefined records model and does extensibility by the side of the cost of manually mapping the design components into the emblematic nodes, which will be after used in the reasoning module. One more limitation of this approach is that it only applies to the very early stages of the design, which be made up of highly accumulated building components and thus will require only minor manual mapping sandwiched between CAD and the symbolic archetypical. This slant will be very inefficient if more detailed design data is required, for example, when the design is in the development stage and a design alternative needs to be evaluated. The third limitation be situated that this model ignores the none-graphic information, which is an unified part of the design papers.

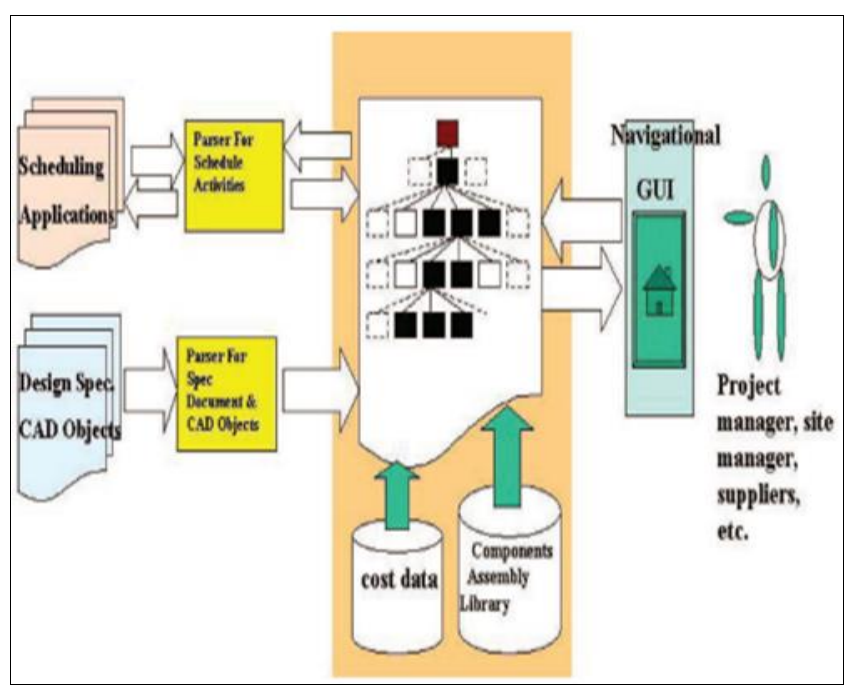

Fig 2: Design and data analysis

\section{Conclusion}

The difficulty of timely salvaging of useful information from diverse data sources is one of the foremost causes that lead to low productivity in the construction engineering. This goal of this research is to afford an approach to handle the data-heterogeneity problems come upon on a construction project. The proposed examination will look at a restructured product model, which binds strategy knowledge, cost data and schedule data together, as a feasible solution for the data combination problem encountered on construction projects. The proposed method uses the knowledge representation of construction projects based on ontology. An original of the proposed archetypal is under increase and will be presented in the near impending.

\section{References}

1. Nambayashi K, Shiino J, Ohtomo T, Uchiiyama Y, Nagayasu O. An Actual State and a Prospect of Computer and Network Application for Transferring and Sharing Information among Participants of Building Construction Viewing from the Job Site in Japan, Proceedings of the Eighth International Conference (ICCBE-VIII), Computing in Civil and Building Engineering, Stanford University, California 2000, 138-153.

2. Pheng LS, Meng CY. Managing Productivity in Construction: JIT Operation and Managements, Ashgate Publishing, Brookfield, Vermont 1997.

3. Sun M, Aouad G, Bakis N, Birchall S, Swan W. Integrated Information Management and Exchange for Water Treatment Projects, Proceedings of the Eighth International Conference (ICCBE-VIII), Computing in Civil and Building Engineering, Stanford University, California 2000, 130-137.

4. O'Brien WJ, Issa RRA, Hammer J, Schmalz M, Guenes J, Bai S. SEEK: Accomplishing Enterprise Integration across Heterogeneous Sources, ITCON-Electronic 
Journal of Information Technology in ConstructionSpecial Edition on Knowledge Management 2000;7(2):101-124.

5. Cims TEEL. CIM steel Integration Standards, Release 2: Overview. Steel Construction Institute, SilWood Park, Ascot, Berkshire 2002, 1.

6. Clayton MJ, Fisher M, Teicholz P, Kunz J. Rapid Conceptual Design Evaluation Using a Virtual Product Model, Engineering Applications of Artificial Intelligence 1996;9(4):439-451.

7. Enkovaara E, Salmi M, Sarja A. RATAS Project, Computer Aided Design for Construction. Building Book, Helsinki, Finland 1988.

8. Pheng LS, Meng CY. Managing Productivity in Construction: JIT Operation and Managements, Ashgate Publishing, Brookfield, Vermont 1997.

9. Rivard H, Fenves SJ. A Representation for Conceptual Design of Buildings, Journal of Computing in Civil Engineering 2000;14(3):151-159.

10. Sun M, Lockley S. Data Exchange System for an Integrated Building Design System, Journal of Automation in Construction 1977;6(2):147-155.

11. Thomas HR, Sanvido VE, Sanders SR. Impact of Material Management on Productivity, Journal of Construction Engineering and Management 1989;115(3):370-384.

12. Staub-French S, Fisher M. Industrial Case Study of Electronic Design, Cost, and Schedule Integration," CIFE Technical Report \#122, Stanford University, Palo Alto, California 2001, 125.

13. Hegazy T, Zaneldin E, Grierson D. Improving Design Coordination for Building Projects. I: Infor 2001. 